\title{
A Local/Global Approach to Mesh Parameterization
}

\section{Citation}

Liu, Ligang, Lei Zhang, Yin Xu, Craig Gotsman, and Steven J. Gortler. 2008. A local/global approach to mesh parameterization. Symposium on Geometry Processing: July 2-4, 2008, Copenhagen, Denmark. Sixth Eurographics/SIGGRAPH symposium on geometry processing. Special Issue, Computer Graphics Forum 27(5): 1495-1504.

\section{Published Version}

http://dx.doi.org/10.1111/j.1467-8659.2008.01290.x

\section{Permanent link}

http://nrs.harvard.edu/urn-3:HUL.InstRepos:2641658

\section{Terms of Use}

This article was downloaded from Harvard University's DASH repository, and is made available under the terms and conditions applicable to Other Posted Material, as set forth at http:// nrs.harvard.edu/urn-3:HUL.InstRepos:dash.current.terms-of-use\#LAA

\section{Share Your Story}

The Harvard community has made this article openly available.

Please share how this access benefits you. Submit a story.

\section{Accessibility}




\title{
A Local/Global Approach to Mesh Parameterization
}

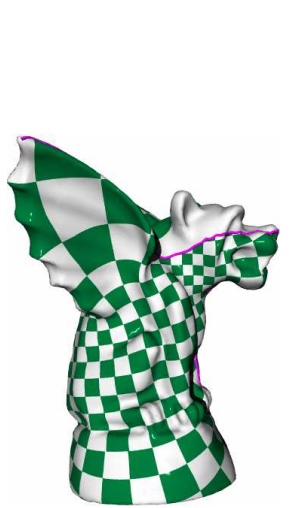

(a) ASAP

\author{
Ligang Liu $^{1 \dagger}$ Lei Zhang $^{1}{\text { Yin } X u^{1} \text { Craig Gotsman }}^{2 \ddagger}$ Steven J. Gortler $^{3 \S}$
}

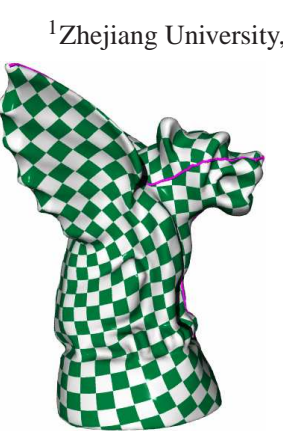

(b) ARAP

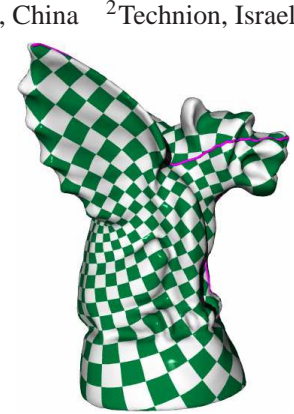

(c) $\mathrm{LABF}$

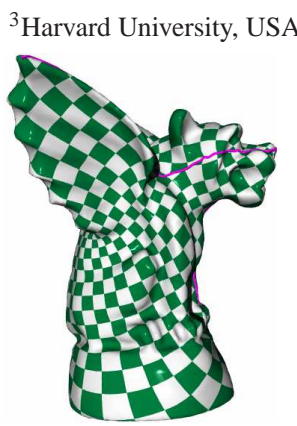

(d) IC

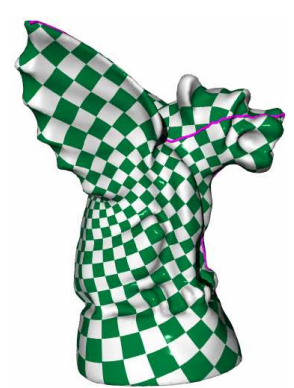

(e) $\mathrm{CP}$

Figure 1: Parameterization of the Gargoyle model using (a) our As-Similar-As-Possible (ASAP) procedure, (b) As-Rigid-As-Possible (ARAP) procedure, (c) Linear ABF [ZLS07], (d) inverse curvature approach [YKL*08], and (e) curvature prescription approach [BCGB08]. The pink lines are the seams of the closed mesh when cut to a disk.

\begin{abstract}
We present a novel approach to parameterize a mesh with disk topology to the plane in a shape-preserving manner. Our key contribution is a local/global algorithm, which combines a local mapping of each $3 D$ triangle to the plane, using transformations taken from a restricted set, with a global "stitch" operation of all triangles, involving a sparse linear system. The local transformations can be taken from a variety offamilies, e.g. similarities or rotations, generating different types of parameterizations. In the first case, the parameterization tries to force each $2 D$ triangle to be an as-similar-as-possible version of its $3 D$ counterpart. This is shown to yield results identical to those of the LSCM algorithm. In the second case, the parameterization tries to force each $2 D$ triangle to be an as-rigid-as-possible version of its $3 D$ counterpart. This approach preserves shape as much as possible. It is simple, effective, and fast, due to pre-factoring of the linear system involved in the global phase. Experimental results show that our approach provides almost isometric parameterizations and obtains more shape-preserving results than other state-of-the-art approaches.
\end{abstract}

We present also a more general "hybrid" parameterization model which provides a continuous spectrum of possibilities, controlled by a single parameter. The two cases described above lie at the two ends of the spectrum. We generalize our local/global algorithm to compute these parameterizations. The local phase may also be accelerated by parallelizing the independent computations per triangle.

Categories and Subject Descriptors (according to ACM CCS): I.3.5 [Computer Graphics]: Computational Geometry and Object Modeling I.3.8 [Computer Graphics]: Application

\section{Introduction}

Surface parameterization of 3D models is an important component in various computer graphics and geometry process-

\footnotetext{
$\dagger$ ligangliu@zju.edu.cn

‡ gotsman@cs.technion.ac.il

$\S$ sjg@cs.harvard.edu
}

ing applications, such as filtering, compression, recognition, texture mapping, and morphing. It involves computing a bijective mapping between a piecewise-linear triangulated surface and a suitable parameter domain. In this paper we consider the parameterization of a surface having the topology of the disk, possibly with boundaries, onto the plane.

In general, the parameterization will incur some metric distortion, since only developable surfaces can be flattened 
onto the plane without any distortion. Hence, the goal of parameterization is to find a bijective mapping which preserves some geometric properties of the original as much as possible, e.g., authalic (area-preserving) mapping, conformal (angle-preserving) mapping, isometric (length-preserving) or some combination of these. Each individual triangle may be easily parameterized without distortion, but then they will no longer fit together properly in the plane.

Inspired by recent work on mesh deformation and modeling [IMH05, SA07], we formulate the parameterization problem as an optimization problem having both local and global elements. In essence, we seek for local transformations which minimize the distortion of each mesh triangle, yet require that they all fit together to a coherent $2 \mathrm{D}$ triangulation. We follow closely the method for "as-rigidas-possible" deformation of triangle meshes described by Sorkine and Alexa [SA07] for mesh editing purposes, and, essentially, apply the same methodology to the problem of mesh parameterization.

\section{Previous Work}

In the past decade, methods for triangular mesh parameterization have been studied extensively. We refer the interested reader to [FH05] and [SPR06] for a survey of the state-ofthe-art in parameterization research.

The linear setting for parameterization offers the advantage of simplicity and validity of parameterization results. Based on Tutte's barycentric mapping theorem [Tut63], Eck et al. [EDD*95] and Floater [Flo97] described a simple approach to parameterization by representing each interior vertex as some convex combination of its neighboring vertices. Depending on the precise weights used, it is possible to achieve a variety of effects, minimizing various distortion measures. The most celebrated weight recipes are the so-called cotangent weights [PP93], and the so-called mean-value weights [Flo03], which are both related to harmonic mappings. Unfortunately, the method of barycentric coordinates requires the boundary of the mesh to be fixed to a convex polygon in the plane, which is somewhat arbitrary, typically resulting in significant distortion. Lee et al. [LKL02] alleviated this somewhat by "padding" the mesh with a virtual boundary, allowing the true boundary to evolve to a less distorted shape. Desbrun et al. [DMA02] were able to generalize the method of barycentric coordinates so that also the boundary vertices are free, subject to socalled "natural" boundary conditions - some additional linear equations. This was shown to be equivalent to the LeastSquares Conformal Mapping (LSCM) method of Levy et al. [LPRM02], which is a least-squares approximation of the discrete Cauchy-Riemann equations, which define continuous conformal mappings. These boundary equations were generalized by Karni et al. [KGG05] to a larger family of barycentric coordinates.

The main problem with linear free-boundary methods is that the parameterization is no longer guaranteed to be bijective, meaning that the resulting $2 \mathrm{D}$ embedding may contain local overlaps (also known as "triangle flips"), global overlaps, or even wind on itself. Karni et al. [KGG05] showed how to solve the more frequent problem of local overlaps in a postprocessing step.

Some parameterization work focuses on directly optimizing the distortion metrics of length, angle or area. These approaches require extensive computation, since the distortion measures are usually highly non-linear. Hormann et al. [HG99] define a deformation-based MIPS energy which requires a non-linear solver. The compute-intensive ABF method of Sheffer et al. [SdS00] computes the parameterization in angle space, with a result minimizing angular distortion. The more efficient ABF++ [SLMB05] and LinABF [ZLS07] have accelerated this method considerably. Other metrics are also used to guide the optimization process for parameterization, such as stretch. These are based on the singular values of the Jacobian matrix of the parameterization mapping [SSGH01], on the Green-Lagrange tensor [MYV93,ZMT05] or the synthesized distortion metric [YYS06].

Other improvements on the method of barycentric coordinates were proposed by Zayer et al. [ZRS05a, ZRS05b] and Yoshizawa et al. [YBS04], who showed how to dynamically adjust the barycentric weights such that the system converges to a parameterization minimizing stretch.

Another approach to parameterization is inspired by recent advances in dimension reduction and manifold learning [LYD*05, ZKK02]. The basic principle is to preserve some geometric property like geodesic distance of a higher dimensional data set while embedding it in a lower dimensional space. For example, Chen et al. [CLZW07] introduce a new parameterization technique based on local tangent space alignment (LTSA), which tries to embed each onering of the mesh in some optimal manner in the plane, and then solves a global linear system to "stitch" the one-rings together to one coherent triangle mesh. In this sense, it is the closest to the approach we describe in this paper.

A series of very recent works on conformal parameterizations by Yang et al. [YKL*08], Ben-Chen et al. [BCGB08] and Springborn et al. [SSP08] manipulate the curvature distribution on a 3D mesh, flattening it by migrating the total curvature so that it is distributed on only a small number of so-called "cone singularities". The other mesh vertices retain no curvature. For the case of a mesh having disk topology with a given boundary, this means concentrating the curvature on the boundary alone. In practice it amounts to computing new lengths for the mesh edges, so that they can be embedded in the plane. Once the 2D edge lengths are computed, the final 2D embedding is computed either by an incremental layout process, or by solving a simple sparse linear system (which happens to be identical to the LSCM process mentioned later in this paper, since that process reproduces 
a planar embedding). The difference between the three methods is the precise algorithm used to manipulate the curvature distribution. These methods, although designed to minimize only conformal (i.e. angular) distortion, in practice produce parameterizations with not too much stretch. They are also relatively easy to compute, thus are strong contenders for use in conformal parameterization scenarios.

\section{Contribution}

We apply the methodology of Sorkine and Alexa [SA07] for mesh editing (which has its origins in a series of papers starting with Sorkine et al. [SCOL $\left.\left.{ }^{*} 04\right]\right)$ to the problem of mesh parameterization. This poses the problem as that of finding optimal local transformations for each individual mesh element from an appropriate family and then "stitching" the transformed triangles together to a 2D mesh. As opposed to [SA07], where the local transformations are applied to one-rings of a vertex and its neighbors, we apply the local transformations to individual triangles. This then becomes a proper finite-element discretization of an associated continuous problem. In this context, our contributions are:

- For the case of local similarity transformations, our method is shown to be equivalent to the well known Least-Squares Conformal Mapping (LSCM) method [LPRM02].

- For the case of local rotational transformations, we provide an efficient and simple iterative "local/global" algorithm to solve the problem. This leads to a parameterization which is close to isometric and shown to be superior to competing algorithms. Additionally, the algorithm minimizes an "intrinsic" deformation energy function that may be expressed in terms of the singular values of the Jacobian of the parameterization, as is the case for many other distortion measures. The algorithm is shown to be very fast, due to pre-factoring of the linear system involved in the global step, and optional parallel processing in the local phase.

- We propose a more general "hybrid" parameterization model which provides a continuous spectrum of possibilities, controlled by a single parameter. The two cases described above (similarities and rotations) lie at the two ends of the spectrum. The hybrid parameterization may also be computed using a similar local/global algorithm.

\section{The General Local/Global Approach}

If each triangle of the $3 \mathrm{D}$ triangle mesh were required to be flattened to the plane independently of the other triangles, this would certainly be easy. Requiring that all the flattened triangles fit together into one mesh with the correct orientations is the main challenge (see Figure 2). Obviously some of the triangles are going to be deformed in the process. Assume we allow each triangle to be deformed by some subset of the 2D linear transformations, in the sense that this transformation "does not count" as a deformation. For example, translations of the triangles are obviously allowed. A conformal parameterization would also allow each triangle to undergo a similarity transformation (only). An isometry would allow only a rotation. An authalic parameterization would allow only transformations with unit determinant.

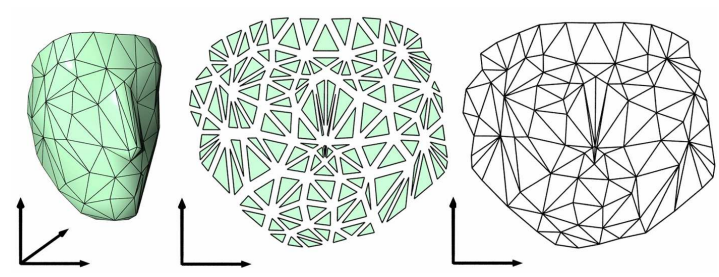

Figure 2: Parameterizing a mesh by aligning locally flattened triangles. (Left) Original 3D mesh; (middle) flattened triangles; (right) 2D parameterization.

Assume the triangles of the 3D triangle mesh are numbered with $t=1$ to $T$ and the area of the 3D triangles are $A_{t}$. Assume that each 3D triangle is equipped with its own local isometric parameterization using a triangle in the plane $x_{t}=\left\{x_{t}^{0}, x_{t}^{1}, x_{t}^{2}\right\}$. Our goal is to find a single parameterization of the entire mesh, i.e., a piecewise linear mapping from the $3 \mathrm{D}$ mesh to the $2 \mathrm{D}$ plane, described by assigning $2 \mathrm{D}$ coordinates $u$ to each of the $n$ vertices. For triangle $t$, let us denote these $2 \mathrm{D}$ coordinates as $u_{t}=\left\{u_{t}^{0}, u_{t}^{1}, u_{t}^{2}\right\}$. Given this setup, the mapping between $x_{t}$ and $u_{t}$ has an associated $2 \times 2 \mathrm{Ja}$ cobian matrix which is constant per triangle. We denote this matrix at triangle $t$ as $J_{t}(u)$ to express its dependence on the $u$. It represents the linear portion of the affine mapping from the triangle described by $x_{t}$ to the triangle described by $u_{t}$. In our method, we will also assign an auxiliary linear transformation $(2 \times 2$ matrix $) L_{t}$ to each triangle taken from some family of allowed transformations $M$ (in particular, we will consider, in turn, $M$ to be the similarity transformations, and later, the rotations).

Define the energy of the parameterization coordinates $u$ and an auxiliary set of $T$ linear transformations $L=$ $\left\{L_{1}, . ., L_{T}\right\}$ to be

$$
E(u, L)=\sum_{t=1}^{T} A_{t}\left\|J_{t}(u)-L_{t}\right\|_{F}^{2}
$$

where $\|\cdot\|_{F}$ is the Frobenius norm. Following Pinkall and Polthier [PP93], this energy may be rewritten in terms of the coordinates $x$ and $u$ (instead of in terms of the Jacobians) in an explicit form (in terms of the mesh vertex coordinates):

$$
\begin{aligned}
& E(u, L) \\
& =\frac{1}{2} \sum_{t=1}^{T} \sum_{i=0}^{2} \cot \left(\theta_{t}^{i}\right)\left\|\left(u_{t}^{i}-u_{t}^{i+1}\right)-L_{t}\left(x_{t}^{i}-x_{t}^{i+1}\right)\right\|^{2}
\end{aligned}
$$

where $\theta_{t}^{i}$ is the angle opposite the edge $\left(x_{t}^{i}, x_{t}^{i+1}\right)$ in the triangle whose vertices are $x_{t}$ and superscripts are all modulo 
2. Note that some of the $u_{t}^{i}$ are identical, as they are shared by more than one triangle in the mesh.

We would like to solve the following optimization problem:

$$
(u, L)=\operatorname{argmin}_{(u, L)} E(u, L) \quad \text { s.t. } L_{t} \in M
$$

Namely, find a set of $n 2 \mathrm{D}$ coordinates $u$ for the mesh vertices and $T$ matrices $L_{1}, . ., L_{T}$ in $M$ such that the Jacobians of the transformation from the given $x$ to the $u$ are closest to the $L_{t}$.

Although we solve for both $u$ and $L$, in the end we are interested only in $u$ while $L$ plays an auxiliary role only. As we shall see below, in many cases, the optimal $u$ may be defined as that minimizing an energy function formulated in terms of the singular values of the Jacobians $J_{t}(u)$. In the next sections, we will examine a number of interesting cases for $M$ and relate our energy functions to those.

\subsection{Best fitting $L$ matrix}

Suppose we are asked to approximate one $2 \times 2$ matrix $J$ as best we can by another $2 \times 2$ matrix $L$, where $L$ is taken from a restricted set of transformations $M$ (we will consider in turn similarities and rotations) and where distance is measured using the Frobenius matrix norm. In other words:

$$
d(J, L)=\|J-L\|_{F}^{2}=\operatorname{tr}\left[(J-L)^{T}(J-L)\right]
$$

This problem can be solved using Procrustes analysis [GD04], and its solution in general is computed using the Singular Value Decomposition (SVD) of $J$.

In particular, using SVD, $J$ may be written as

$$
J=U \Sigma V^{T}
$$

where $U$ and $V$ are orthonormal, and $\Sigma$ is a diagonal matrix:

$$
\Sigma=\left(\begin{array}{cc}
\sigma_{1} & 0 \\
0 & \sigma_{2}
\end{array}\right)
$$

We use here a "signed version" of the SVD, where the determinant of $U V^{T}$ is constrained to be positive, $\sigma_{1}$ is positive and $\sigma_{2}$ may be positive or negative. We refer to these $\sigma$ as signed singular values. This signed version is needed to constrain our Procrustes solutions to exclude orientation reversing transformations.

Given this decomposition, it is easy to show that the optimal rotation minimizing the distance $d(J, L)$ is obtained by setting both singular values to 1 , i.e. $L=U V^{T}$. Similarly, the optimal similarity transform is obtained by setting both singular values to $\left(\sigma_{1}+\sigma_{2}\right) / 2$. See Figure 3 for examples.

\subsection{As-similar-as-possible (ASAP) mappings}

Conformal mappings are those which preserve angles, which are invariant under similarity transformations. Thus, in order to produce a conformal-type parameterization, the family
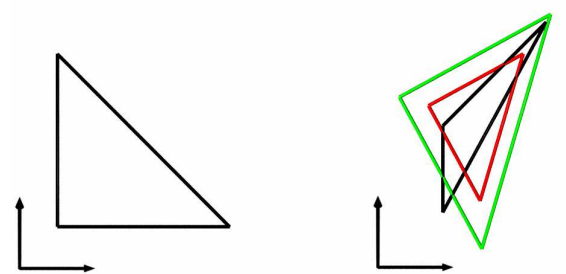

Figure 3: Optimal Procrustes transformations: Left: source triangle; Right: target triangle in black, optimal similarity of source in red, optimal rotated version of source in green.

$M$ of allowed transformations should be similarities, which may be parameterized as all matrices of the form:

$$
M=\left\{\left(\begin{array}{cc}
a & b \\
-b & a
\end{array}\right): a, b \in \mathbf{R}\right\}
$$

Thus we may represent the allowed $L_{t}$ in the energy function (2) with the variables $a=\left(a_{1}, . ., a_{T}\right), b=\left(b_{1}, . ., b_{T}\right)$. Since the $x_{t}^{i}$ and $\theta_{t}^{i}$ are fixed, the energy function is quadratic in the variables $a, b, u$ and thus may be minimized by solving a large sparse linear system with these variables.

Since we try to stay close to the family of similarity transformations, we call this parameterization "as-similaras-possible" (ASAP). Appendix A proves that solving (2) with this $M$ is equivalent to finding the $u$ that minimizes Lévy's conformal energy:

$$
\sum_{t=1}^{T} A_{t}\left(\sigma_{1, t}-\sigma_{2, t}\right)^{2}
$$

where $\sigma_{1, t}$ and $\sigma_{2, t}$ are the signed singular values of $J_{t}$ - the Jacobian of the $t$-th triangle's transformation. Since the Least-Square Conformal mapping (LSCM) technique [LPRM02] also minimizes precisely this energy, the two techniques are equivalent.

As with LSCM, for non-developable meshes, the (trivial) solution that collapses all of the vertices to a single point in the plane achieves a global minimum - zero energy. This can be avoided by constraining two vertices to two different locations in the plane. In practice, we pin down the two vertices most distant from each other (the diameter) in the mesh.

\subsection{As-rigid-as-possible (ARAP) mappings}

While conformal mappings have many nice mathematical properties, they are not always exactly what the application needs. The fact that arbitrary scaling factors may creep into the parameterization makes it unsuitable for applications which try to minimize "stretch" and preserve the proportions of the triangles.

To obtain an "as-rigid-as-possible" mapping we limit the 
family of allowed local transformations to be just rotations:

$$
M=\left\{\left(\begin{array}{cc}
\cos \theta & \sin \theta \\
-\sin \theta & \cos \theta
\end{array}\right): \theta \in[0,2 \pi)\right\}
$$

Or, in other words, the same as (3), only with the extra requirement that $a^{2}+b^{2}=1$.

Appendix A proves that solving (2) with this $M$ is equivalent to finding the $u$ which minimizes

$$
\sum_{t=1}^{T} A_{t}\left[\left(\sigma_{1, t}-1\right)^{2}+\left(\sigma_{2, t}-1\right)^{2}\right]
$$

This energy is similar to the Green-Lagrange energy [MYV93, ZMT05], which uses terms of the form $\left[\left(\sigma_{1, t}^{2}-1\right)^{2}+\left(\sigma_{2, t}^{2}-1\right)^{2}\right]$ and also produces parameterizations which are close to isometric.

Alas, the extra condition on $L_{t}$ in (1) means that the energy function may no longer be minimized by solving a linear system.

\subsection{Local/Global Algorithm}

To solve the minimization problem (2) for an ARAP mapping, we adapt the local/global algorithm of [SA07]. This iterates between two phases. In the first local phase, the optimal rotation $L_{t}$ is computed per triangle, assuming the $u$ are fixed. Then, in the second global phase, the $L_{t}$ are assumed fixed, and the optimal $u$ are solved for as a sparse linear system. (Recall that $x_{t}$ are fixed throughout the algorithm.) Since each step is guaranteed to reduce the energy, this energy will eventually converge. Additionally, since the matrix of the global phase is unchanged from iteration to iteration, it only has to be factored once and reused at each iteration.

\subsubsection{Local Phase}

The local phase can be solved using the SVD factorization of $J$ as described in Section 4.1. Equivalently, and analogously to [SA07], for ARAP one can also perform the SVD factorization directly on the following "cross-covariance" matrix in place of $J_{t}(u)$

$$
S_{t}(u)=\sum_{i=0}^{2} \cot \left(\theta_{t}^{i}\right)\left(u_{t}^{i}-u_{t}^{i+1}\right)\left(x_{t}^{i}-x_{t}^{i+1}\right)^{T}
$$

\subsubsection{Global Phase}

For fixed $L_{t}$, the energy $E(u, L)$ is quadratic in $u$. The minimum $u$ can be found by setting the gradients of (1) to zero and solving the associated linear system. To calculate this, overloading the notation slightly, we rewrite the energy function in terms of the mesh half-edges:

$$
\begin{aligned}
& E(u, L) \\
& =\frac{1}{2} \sum_{t=1}^{T} \sum_{i=0}^{2} \cot \left(\theta_{t}^{i}\right)\left\|\left(u_{t}^{i}-u_{t}^{i+1}\right)-L_{t}\left(x_{t}^{i}-x_{t}^{i+1}\right)\right\|^{2} \\
& =\frac{1}{2} \sum_{(i, j) \in h e} \cot \left(\theta_{i j}\right)\left\|\left(u_{i}-u_{j}\right)-L_{t(i, j)}\left(x_{i}-x_{j}\right)\right\|^{2}
\end{aligned}
$$

where $h e$ is the set of half-edges in the mesh, $u_{i}$ and $x_{i}$ are coordinates of vertices $i, t(i, j)$ is the triangle containing the half-edge $(i, j)$, and $\theta_{i j}$ is the angle opposite $(i, j)$ in $t(i, j)$.

Setting the gradient to zero, we obtain the following set of linear equations for $u$.

$$
\begin{array}{r}
\sum_{j \in N(i)}\left[\cot \left(\theta_{i j}\right)+\cot \left(\theta_{j i}\right)\right]\left(u_{i}-u_{j}\right) \\
=\sum_{j \in N(i)}\left[\cot \left(\theta_{i j}\right) L_{t(i, j)}+\cot \left(\theta_{j i}\right) L_{t(j, i)}\right]\left(x_{i}-x_{j}\right), \\
\forall i=1, . ., n .
\end{array}
$$

The entries of the associated matrix depend only on the geometry of the input 3D mesh. Thus this sparse matrix is fixed throughout the algorithm, allowing us to pre-factor it (e.g. with Cholesky decomposition) [GvL05, In0] and reuse the factorization many times throughout the algorithm in order to accelerate the process. This has a significant impact on algorithm efficiency.

Unfortunately, stitching the triangles using a global Poisson equation may result in some triangles "flipping" their orientation especially for a highly curved surface with compact boundary. We solve this with a final post-processing phase, e.g., the "convex virtual boundary" algorithm of Karni et al. [KGG05]. Since in most cases, there are only a few flips, sprinkled throughout the parameterization, the postprocessing solves the flips without changing much else.

\subsection{The initial parameterization}

Our local/global algorithm requires an initial parameterization $\mathbf{M}$ to start it off. The basic requirement from the initial parameterization is that it be a valid embedding (contain no flips) reasonably close to a parameterization with not too much distortion, and be fast to generate. Candidates are the shape-preserving method [Flo97] and LSCM [LPRM02] as they can be computed quickly. We found the shapepreserving parameterization more suitable for meshes with one boundary, and LSCM [LPRM02] for meshes with multiple boundaries. The experimental results shown in Section 6 are obtained using these initial parameterizations.

We tested the sensitivity of our algorithm to different types of M's, as mentioned above. We found that the algorithm is not sensitive to $\mathbf{M}$ at all. Figure 4 shows the progress of the iterative algorithm when initialized with the shapepreserving parameterization and LSCM. Both converge fast and stably. 

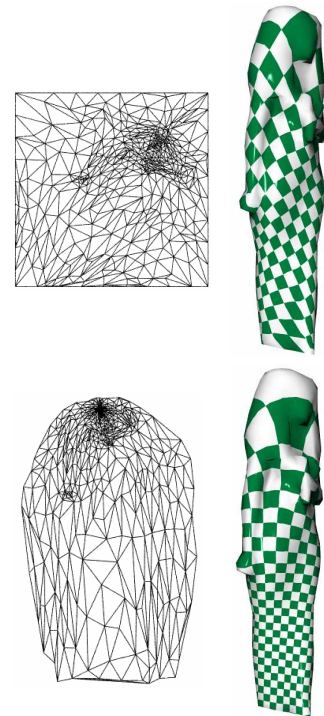

Initial guess
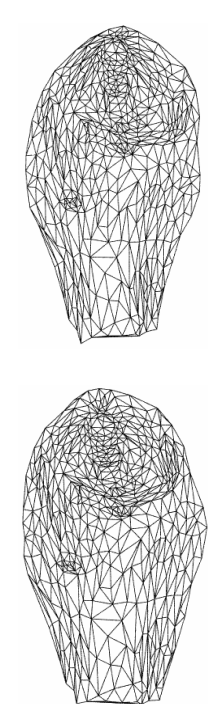

1 Iteration
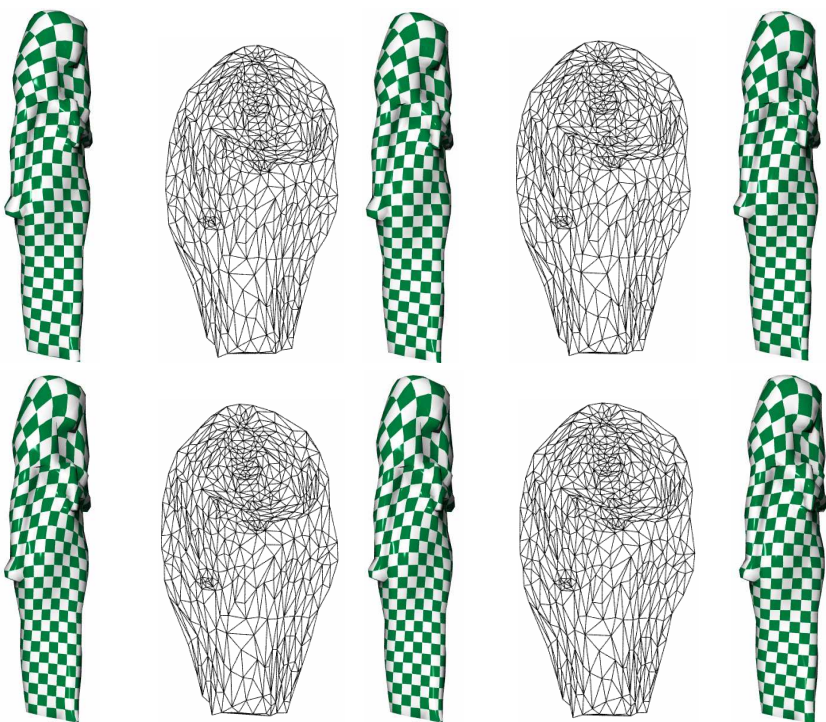

3 Iterations

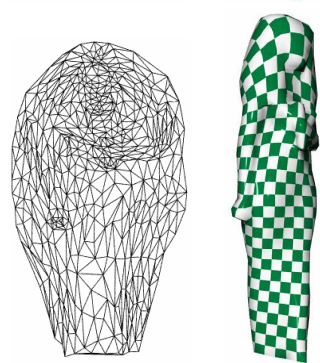

Final result

Figure 4: Successive iterations of the local/global ARAP algorithm. (Top) Initialized using Floater's shape-preserving parameterization [Flo97]. (Bottom) Lower row: Initialized using LSCM parameterization [LPRM02].

\section{The Hybrid Model}

Our ASAP parameterization belongs to the family of (approximately) conformal maps and may be computed easily by solving a simple linear system. However, it is not the most area-preserving among the conformal parameterizations, and, in fact, the non-linear $\mathrm{ABF}++$ method preserves area much better, while still being approximately conformal. On the other hand, our ARAP parameterization preserves areas much better, but since it it strives to be isometric, it might damage the conformality in this effort. We now present an energy function which is a generalization of (1), which provides a means to generate a parameterization anywhere between ASAP and ARAP. The two latter are endpoints of the spectrum, and the result is controlled by a parameter $\lambda \in[0, \infty)$.

The hybrid energy function is

$$
\begin{aligned}
& E(u, a, b)= \\
& \frac{1}{2} \sum_{t=1}^{T}\left[\sum_{i=0}^{2} \cot \left(\theta_{t}^{i}\right)\left\|\nabla e_{t}^{i}\right\|^{2}+\lambda\left(a_{t}^{2}+b_{t}^{2}-1\right)^{2}\right],
\end{aligned}
$$

where

$$
\nabla e_{t}^{i}=\left(u_{t}^{i}-u_{t}^{i+1}\right)-\left(\begin{array}{cc}
a_{t} & b_{t} \\
-b_{t} & a_{t}
\end{array}\right)\left(x_{t}^{i}-x_{t}^{i+1}\right) .
$$

Setting $\lambda=0$ will be equivalent to ASAP while a very large value of $\lambda$ will be equivalent to ARAP. Any value inbetween will yield an intermediate parameterization, so the user may control the tradeoff between conformality and area-preservation.
Solving for the parameterization coordinates $u$ which minimize $E(u, a, b)$ involves solving also for the auxiliary vectors of unknowns of the similarity transformations $a$ and $b$. The value of $\lambda$ indicates how much we want to force the similarity to be a rotation. A local/global algorithm similar to that we use for solving for the ARAP parameterization may be used here as well, i.e. iterate while alternating between two phases: one local and one global. Recall that $x$ is fixed (derived directly from the input 3D mesh). The local phase keeps the parameterization coordinates $u$ fixed and solves for the optimal $a_{t}$ and $b_{t}$ per triangle $t$. Examination of (5) reveals that this involves solving two cubic equations in $a_{t}$ and $b_{t}$. Furthermore, this reduces to a single cubic equation in $a_{t}$, (Equation (B3) in Appendix B), which may be solved analytically. The global phase keeps both vectors $a$ and $b$ fixed and solves a global sparse linear system (similar to (4)) for $u$. Since the matrix of the linear system is fixed throughout all iterations, it may be pre-factored at the beginning, and reused in all iterations thereafter. Thus the runtime of the procedure is dominated by the first iteration. This makes for a simple and efficient algorithm.

\section{Experimental Results and Comparison}

We have applied our approach to parameterize a variety of 3D meshes and compared with other relevant methods. These include LSCM (equivalent to our ASAP method) [LPRM02], direct $\mathrm{ABF}++(\mathrm{ABF}++$ without the hierarchical solver) [SLMB05], which we label $\mathrm{dABF}++$, linear $\mathrm{ABF}$ [ZLS07], which we label LABF, inverse curvature 

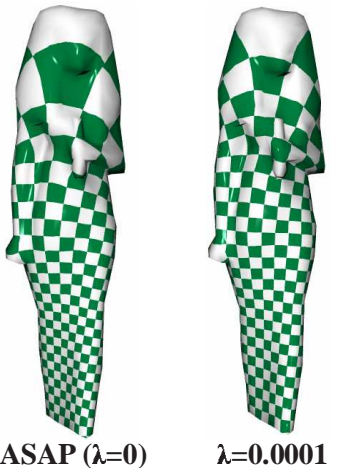

$(2.05,15.4)$

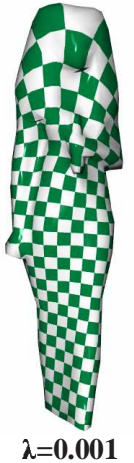

$(2.07,2.88)$

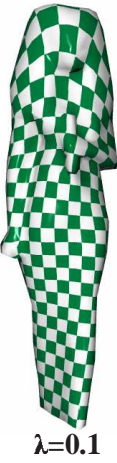

$(2.18,2.14)$

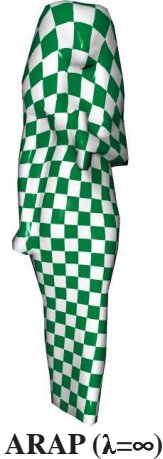

$(2.19,2.11)$

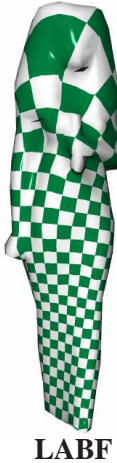

$(2.12,9.12)$
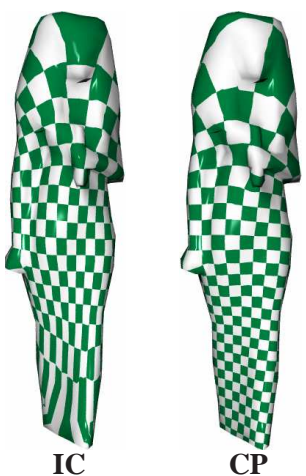

$(3.09,3.91)$

$(2.29,11.9)$

Figure 5: Isis model: Effect of $\lambda$ in hybrid energy function (5) compared with results of other algorithms. Numbers in brackets denote angular distortion and area distortion.

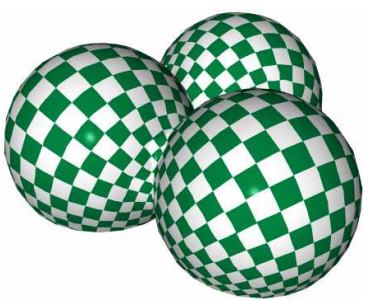

$\operatorname{ASAP}(\lambda=0) \quad(2.02,2.42)$

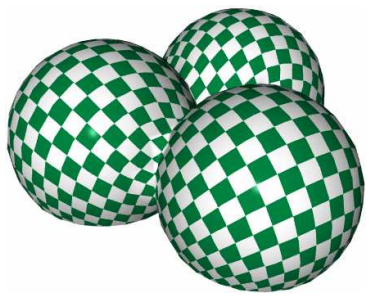

$\operatorname{ARAP}(\lambda=\infty)(2.07,2.04)$

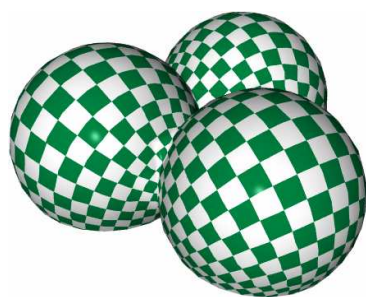

$\lambda=\mathbf{1 0}^{-4}(2.02,2.31)$

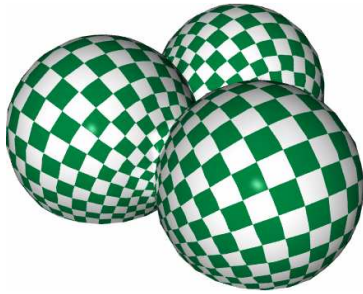

LABF $(2.05,2.34)$

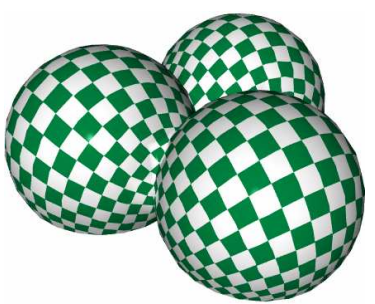

$\lambda=\mathbf{1 0}^{-3} \quad(2.03,2.15)$

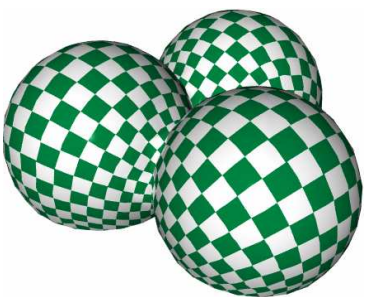

IC $(2.06,2.42)$

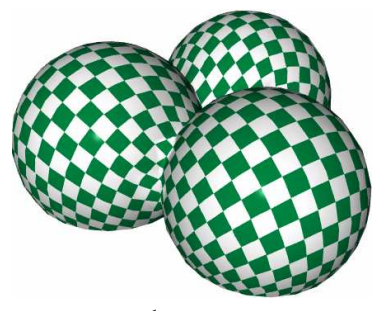

$\lambda=\mathbf{1 0}^{-1}(2.07,2.05)$

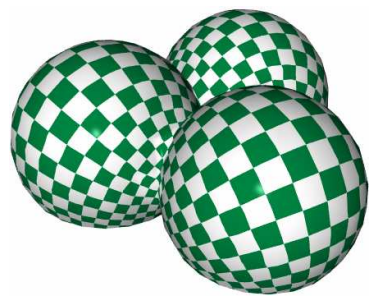

$\mathbf{C P}(2.36,3.18)$

Figure 6: Balls model: Effect of $\lambda$ in hybrid energy function (5) compared with results of other algorithms. Numbers in brackets denote angular distortion and area distortion.

[YKL ${ }^{*} 08$ ], which we label IC, and curvature prescription [BCGB08], which we label CP. We show some results in Fig. 1 and Figs. 5-8. In our algorithm, we used the sparse Cholesky linear solver [In0] for the global systems and the analytic solution to Equation (B3) for the local systems. The IC results were kindly provided by Yang and the $\mathrm{CP}$ results by Ben-Chen, the authors of those methods, who ran their own software. The results of LABF where obtained by running software kindly provided by Zayer.

Computing the ASAP parameterization involves the solution of one sparse linear system, thus is very fast. Computing ARAP involves running the local/global algorithm. This converged in up to 10 iterations in all our experiments. Computing the hybrid also involves an iterative local/global algo- rithm, but with the local phase using the analytic solution to Equation (B3) instead of a simple $2 \times 2$ SVD operation.

The figures show the parameterizations resulting from ASAP, ARAP and the hybrid method with some interesting values of $\lambda$. These are compared with the results of the other algorithms. The runtime of our local/global procedure is comparable to the state-of-the-art $\mathrm{ABF}++$. Our local/global parameterization method may be applied also to meshes with multiple boundaries. Fig. 8 shows one such result. The LABF method is not applicable to such inputs.

To quantify the parameterization distortion, we compute both the angle and area distortion metric defined using the signed singular values $\sigma_{1, t}$ and $\sigma_{2, t}$ of the Jacobians $J_{t}$ for 


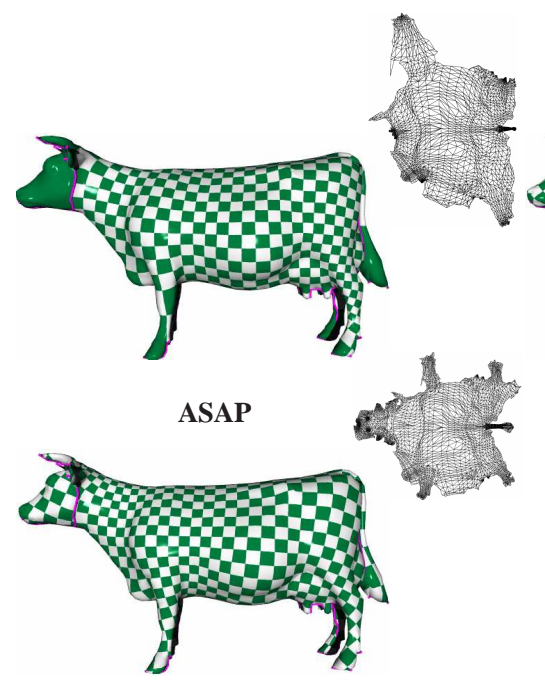

LABF

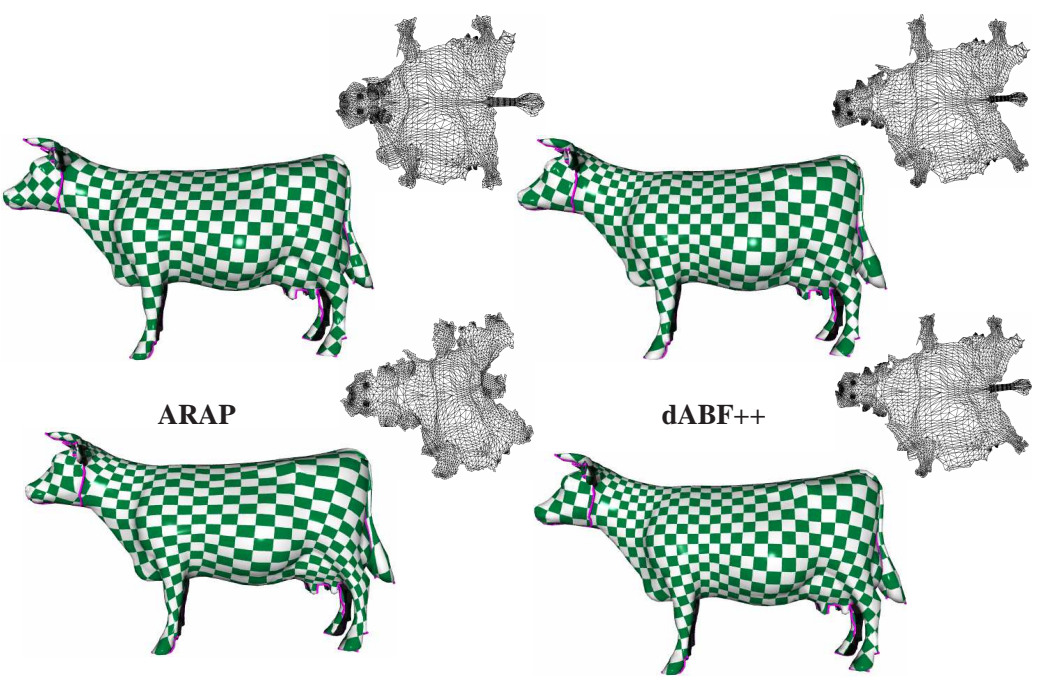

CP

Figure 7: Cow model: Comparison with other algorithms. The pink lines are the seams of the closed mesh when cut to a disk. Triangulations are the $2 D$ parameterizations.

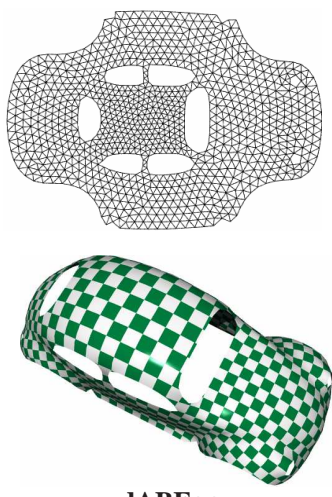

dABF++

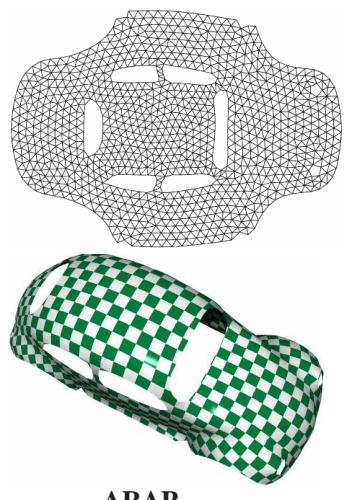

ARAP

Figure 8: Beetle model with multiple boundaries: (Left) Direct $A B F++$. (Right) ARAP.

each triangle, as defined in [HG99, DMK03]:

$$
\begin{aligned}
D^{\text {angle }} & =\sum_{t} \rho_{t}\left(\sigma_{t}^{1} / \sigma_{t}^{2}+\sigma_{t}^{2} / \sigma_{t}^{1}\right) \\
D^{\text {area }} & =\sum_{t} \rho_{t}\left(\sigma_{t}^{1} \sigma_{t}^{2}+1 /\left(\sigma_{t}^{1} \sigma_{t}^{2}\right)\right)
\end{aligned}
$$

where the weight $\rho_{t}$ is

$$
\rho_{t}=A_{t} / \sum_{t} A_{t}
$$

and $A_{t}$ is the area of triangle $t$. The values of the distortion measures obtained by the various algorithms is summarized in Table 1. As is evident in the table, our ARAP parameterization consistently gives the best value of $D^{\text {area }}$, at a very

small, even insignificant, penalty in $D^{\text {angle }}$. It is possible to significantly improve the value of $D^{\text {area }}$ relative to LSCM even by introducing a small value of $\lambda$.

\section{Discussion and Conclusion}

We have presented a novel approach to parameterization of 3D mesh surfaces, by minimizing a very general energy function. We have also provided a simple and efficient lo$\mathrm{cal} / \mathrm{global}$ algorithm for computing these parameterizations. The local component of the algorithm tries to minimize distortion of the individual 2D triangles relative to the original 3D mesh geometry, while the global component guarantees that the resulting $2 \mathrm{D}$ triangles fit together in a coherent manner. Both phases may be computed efficiently, the local one taking advantage of parallelism (possibly on the GPU), and the global one taking advantage of efficient sparse linear solvers with factorization.

We have shown that our ASAP definition is equivalent to LSCM, while ARAP can yield more shape-preserving results compared with other contemporary methods. A hybrid model, coupled with a similar efficient local/global algorithm, allows to obtain parameterizations anywhere inbetween these two. While we have applied this method to parameterizing meshes with disk topology only, it seems it could be possible to use the same methodology for higher genus meshes, possibly through the use of one-forms. Positional constraints on some of the vertices in parameter space can also be imposed without complicating the algorithm, as these types of (hard or soft) constraints may be easily incorporated into the energy functions we optimize. 
Table 1: Comparison of distortion measures of different parameterization methods.

\begin{tabular}{|c|c|c|c|c|c|c|c|c|c|c|c|c|}
\hline \multirow{2}{*}{ Model } & \multicolumn{6}{|c|}{$D^{\text {angle }}$} & \multicolumn{6}{|c|}{$D^{\text {area }}$} \\
\hline & LSCM & $\mathrm{dABF}$ & LABF & IC & $\mathrm{CP}$ & ARAP & LSCM & dABF & LABF & IC & $\mathrm{CP}$ & ARAP \\
\hline Gargoyle & 2.00 & 2.00 & 2.00 & 2.05 & 2.00 & 2.06 & 88.14 & 2.64 & 2.64 & 2.67 & 2.64 & 2.05 \\
\hline Isis & 2.05 & 2.08 & 2.12 & 3.09 & 2.29 & 2.19 & 15.36 & 8.37 & 9.12 & 3.91 & 11.94 & 2.11 \\
\hline Balls & 2.02 & 2.06 & 2.05 & 2.06 & 2.36 & 2.07 & 2.40 & 2.35 & 2.34 & 2.42 & 3.18 & 2.04 \\
\hline Cow & 2.01 & 2.01 & 2.01 & 2.46 & 2.01 & 2.03 & 30.09 & 2.19 & 2.22 & 2.51 & 2.18 & 2.03 \\
\hline Beetle & 2.00 & 2.00 & - & 2.02 & 2.00 & 2.01 & 2.08 & 2.09 & - & 2.14 & 2.10 & 2.01 \\
\hline
\end{tabular}

Our local/global algorithm embodies the motif "think globally, act locally", which is emerging as a powerful technique in geometry processing. This paper and others [DG07] have applied this to the problem of mesh parameterization and future work will address other applications where the technique may also be very effective.

\section{Acknowledgements}

Thanks to Yongliang Yang and Miri Ben-Chen for providing results of the algorithms of [YKL*08] and [BCGB08]. Thanks also to Hugues Hoppe for his many helpful insights on this topic. This work is supported by National Natural Science Foundation of China (grants \# 60776799, 60503067). Craig Gotsman and Steven Gortler were partially supported by United States - Israel Binational Science Foundation grant \# 2006089.

\section{References}

[BCGB08] Ben-Chen M., Gotsman C., Bunin G.: Conformal flattening by curvature prescription and metric scaling. Computer Graphics Forum 27, 2 (2008), 449-458. (Proc. Eurographics 2008). 2, 7, 9

[CLZW07] Chen Z. G., LIU L. G., Zhang Z. Y., WANG G. J.: Surface parameterization via aligning optimal local flattening. In Proc. Symposium on Solid and Physical Modeling (2007), pp. 291-296. 2

[DG07] Dong S., GARLAND M.: Iterative methods for improving mesh parameterizations. In Proc. IEEE Shape Modeling International (2007), pp. 119-210. 9

[DMA02] Desbrun M., Meyer M., Alliez P.: Intrinsic parameterization of surface meshes. Computer Graphics Forum 21, 3 (2002), C209-C218. (Proc. Eurographics'02). 2

[DMK03] Degener P., Meseth J., Klein R.: An adaptable surface parameterization method. In Proc. of 12th International Meshing Roundtable (2003), pp. 227-237. 8

[EDD*95] Eck M., DeRose T., Duchamp T., Hoppe H., LOUNSBERY M., STUETZLE W.: Multiresolution analysis of arbitrary meshes. In Proc. Siggraph'95 (1995), pp. 173-182. 2

[FH05] Floater M. S., Hormann K.: Surface parameterization: a tutorial and survey. In Advances in Multiresolution for Geometric Modeling (2005), pp. 157-186. 2

[Flo97] FLOATER M. S.: Parameterization and smooth approximation of surface triangulations. Computer Aided Geometry Design 14 (1997), 231-250. 2, 5
[Flo03] FloATER M.: Mean value coordinates. Computer Aided Geometric Design 20, 1 (2003), 19-27. 2

[GD04] Gower J. C., Dijksterhuis G. B.: Procrustes Problems. Oxford University Press, 2004. 4

[GvL05] Golub G. H., van LoAn C. F.: Matrix Computation. Johns Hopkins Studies in Mathematical Sciences, 2005. 5

[HG99] HORMANN K., GREINER G.: MIPS: an efficient global parameterization method. In Proc. Curves and Surfaces (1999), pp. $153-162.2,8$

[IMH05] Igarashi T., Moscovich T., Hughes J. F.: Asrigid-as-possible shape manipulation. ACM TOG 24, 3 (2005), C1134-C1141. (Proc. Siggraph'05). 2

[In0] Intel math kernel library. http://developer.intel.com. 5,7

[KGG05] Karni Z., Gotsman C., Gortler S. J.: Freeboundary linear parameterization of $3 \mathrm{D}$ meshes in the presence of constraints. In Proc. IEEE Shape Modeling and Applications (2005), pp. 268-277. 2, 5

[LKL02] LEE Y., KIM H. S., LEE S.: Mesh parameterization with a virtual boundary. Computer and Graphics 26, 5 (2002), 677-686. 2

[LPRM02] LÉvy B., Petitjean S., Ray N., Maillot J.: Least squares conformal maps for automatic texture atlas generation. ACM TOG 21, 3 (2002), C362-C371. (Proc. Siggraph'02). $2,3,4,5,6,10$

[LYD*05] LIU Y. S., YU P. Q., Du M. C., Yong J. H., ZhanG H., PAUl J. C.: Mesh parameterization for an open connected surface without partition. In Proc. of Computer Aided Design and Computer Graphics (2005), pp. 306-312. 2

[MYV93] Maillot J., Yahia H., Verroust A.: Interactive texture mapping. In Proc. Siggraph'93 (1993), pp. 27-34. 2, 5

[PP93] Pinkall U., Polthier K.: Computing discrete minimal surface and their conjugates. Experimental Mathematics 2, 1 (1993), 15-36. 2, 3

[SA07] Sorkine O., AleXa M.: As-rigid-as-possible surface modeling. In Proc. Eurographics Symposium on Geometry Processing (2007), pp. 109-116. 2, 3, 5

[SCOL*04] Sorkine O., Cohen-Or D., Lipman Y., Alexa M., Roessl C., SEIDEL H.-P.: Laplacian surface editing. In Proc. Eurographics Symposium on Geometry Processing (2004), pp. $175-184.3$

[SdS00] ShefFer A., DE Sturler E.: Parameterization of faceted surfaces for meshing using angle-based flattening. Engineering with Computer 17, 3 (2000), 326-337. 2 
[SLMB05] SHEFFer A., LÉVy B., MOgILnitsky M., BoGOMYAKOV A.: ABF++: Fast and robust angle-based flattening. ACM TOG 24, 2 (2005), 311-330. 2, 6

[SPR06] SheFFer A., Praun E., Rose K.: Mesh parameterization methods and their applications. Foundation and Trends in Computer Graphics and Vision 2, 2 (2006), 105-171. 2

[SSGH01] Sander P. V., Snyder J., Gorter S. J., Hoppe H.: Texture mapping progressive meshes. In Proc. SIGGRAPH '01 (2001), pp. 409-416. 2

[SSP08] Springborn B., Schroeder P., Pinkall U.: Conformal equivalence of triangle meshes. ACM Transactions on Graphics 27, 3 (2008). (Proc. SIGGRAPH 2008). 2

[Tut63] TutTe W.: How to draw a graph. In Proc. London Mathematical Society (1963), pp. 743-768. 2

[YBS04] Yoshizawa S., Belyaev A. G., Seidel H.-P.: A fast and simple stretch-minimizing mesh parameterization. In Proc. Shape Modeling International (2004), pp. 200-208. 2

[YKL*08] Yang Y., Kim J., Luo F., Hu S., Gu X.: Optimal surface parameterization using inverse curvature map. IEEE TVCG (2008). To appear. 2, 7, 9

[YYS06] YAN J. Q., YANG X., SHI P. F.: Mesh parameterization by minimizing the synthesized distortion metric with the coefficient optimizing algorithm. IEEE Transactions on Visualization and Computer Graphics 12, 1 (2006), 83-92. 2

[ZKK02] Zigelman G., Kimmel R., KiRyati N.: Texture mapping using surface flattening via multidimensional scaling. IEEE Transactions on Visualization and Computer Graphics 8, 2 (2002), 198-207. 2

[ZLS07] ZaYer R., LÉvy B., SeIdel H.-P.: Linear angle based parameterization. In Proc. Eurographics Symposium on Geometry Processing (2007), pp. 135-141. 2, 6

[ZMT05] Zhang E., Mischaikow K., Turk G.: Featurebased surface parameterization and texture mapping. ACM TOG 24,1 (2005), 1-27. 2, 5

[ZRS05a] ZAYer R., Roessl C., Seidel H.-P.: Discrete tensorial quasi-harmonic maps. In Proc. IEEE Shape Modeling and Applications (2005), pp. 278-287. 2

[ZRS05b] Zayer R., Roessl C., Seidel H.-P.: Setting the boundary free: A composite approach to surface parameterization. In Proc. Eurographics Symposium on Geometry Processing (2005), pp. 91-100. 2

\section{Appendix A:}

Consider minimizing the following energy function

$$
\begin{aligned}
E(u, L) & =\sum_{t=1}^{T} A_{t}\left\|J_{t}(u)-L_{t}\right\|_{F}^{2} \\
& =\sum_{t=1}^{T} A_{t} \operatorname{tr}\left(\left(J_{t}(u)-L_{t}\right)^{T}\left(J_{t}(u)-L_{t}\right)\right)
\end{aligned}
$$

subject to $L_{t} \in M$.

If we define $E(u)=\min _{L} E(u, L)$, then our optimization becomes $\min _{u} E(u)$. To understand $E(u)$, we use the fact that for a fixed $u$, the best $L_{t}$ for each triangle can be expressed using Procrustes analysis. In particular, if by the signed SVD, $J_{t}=U_{t} \Sigma_{t} V_{t}^{T}$, for $\Sigma_{t}=$ $\left(\begin{array}{cc}\sigma_{1, t} & 0 \\ 0 & \sigma_{2, t}\end{array}\right)$, then the optimal $L_{t}$ is of the form $L_{t}=U_{t} K_{t} V_{t}^{T}$, for $K_{t}=\left(\begin{array}{cc}k_{1, t} & 0 \\ 0 & k_{2, t}\end{array}\right)$ where for the ARAP case, $k_{1, t}=k_{2, t}=1$, and for the ASAP case, $k_{1, t}=k_{2, t}=\left(\sigma_{1, t}+\sigma_{2, t}\right) / 2$.

Substituting this in (A1), we get

$$
\begin{aligned}
& E(u) \\
& =\sum_{t=1}^{T} A_{t} \operatorname{tr}\left(\left(U_{t} \Sigma_{t} V_{t}^{T}-U_{t} K_{t} V_{t}^{T}\right)^{T}\left(U_{t} \Sigma_{t} V_{t}^{T}-U_{t} K_{t} V_{t}^{T}\right)\right) \\
& =\sum_{t=1}^{T} A_{t} \operatorname{tr}\left(V_{t} \Sigma_{t}^{2} V_{t}^{T}-2 V_{t} \Sigma_{t} K_{t} V_{t}^{T}+V_{t} K_{t}^{2} V_{t}^{T}\right) \\
& =\sum_{t=1}^{T} A_{t} \operatorname{tr}\left(\Sigma_{t}^{2}-2 \Sigma_{t} K_{t}+K_{t}^{2}\right) \\
& =\sum_{t=1}^{T} A_{t}\left[\left(\sigma_{1, t}-k_{1, t}\right)^{2}+\left(\sigma_{2, t}-k_{2, t}\right)^{2}\right]
\end{aligned}
$$

In particular, for the ARAP case, $k_{1, t}=k_{2, t}=1$, so

$$
E(u)=\sum_{t=1}^{T} A_{t}\left[\left(\sigma_{1, t}-1\right)^{2}+\left(\sigma_{2, t}-1\right)^{2}\right] .
$$

and for the ASAP case, $k_{1, t}=k_{2, t}=\left(\sigma_{1, t}+\sigma_{2, t}\right) / 2$, so

$$
E(u)=\sum_{t=1}^{T} A_{t}\left(\sigma_{1, t}-\sigma_{2, t}\right)^{2},
$$

which has been shown to be identical to Lévy's conformal energy [LPRM02], minimized by the LSCM algorithm.

\section{Appendix B:}

Assume we seek $a$ and $b$ minimizing

$$
E(a, b)=\sum_{i=0}^{2} w_{i}\left\|\nabla e^{i}\right\|^{2}+\lambda\left(a^{2}+b^{2}-1\right)^{2}
$$

where $\nabla e^{i}=\left(u^{i}-u^{i+1}\right)-\left(\begin{array}{cc}a & b \\ -b & a\end{array}\right)\left(v^{i}-v^{i+1}\right)$ and all other quantities are constants.

$$
\text { Denote } u^{i}-u^{i+1}=\left(\begin{array}{c}
\nabla u_{x}^{i} \\
\nabla u_{y}^{i}
\end{array}\right), v^{i}-v^{i+1}=\left(\begin{array}{c}
\nabla v_{x}^{i} \\
\nabla v_{y}^{i}
\end{array}\right) \text {. Taking }
$$
$\partial E / \partial a=0$ and $\partial E / \partial b=0$ yields:

$$
\begin{aligned}
& C_{1} a+2 \lambda a\left(a^{2}+b^{2}-1\right)=C_{2} \\
& C_{1} b+2 \lambda b\left(a^{2}+b^{2}-1\right)=C_{3}
\end{aligned}
$$

where $\quad C_{1}=\sum_{i=0}^{2} w_{i}\left[\left(\nabla v_{x}^{i}\right)^{2}+\left(\nabla v_{y}^{i}\right)^{2}\right]$, $C_{2} \quad=\quad \sum_{i=0}^{2} w_{i}\left[\nabla u_{x}^{i} \nabla v_{x}^{i}+\nabla u_{y}^{i} \nabla v_{y}^{i}\right], \quad C_{3}=$ $\sum_{i=0}^{2} w_{i}\left[\nabla u_{x}^{i} \nabla v_{y}^{i}-\nabla u_{y}^{i} \nabla v_{x}^{i}\right]$.

Dividing (B1) by (B2) gives $b=\left(C_{3} / C_{2}\right) a$, and the final cubic equation in $a$ :

$$
\frac{2 \lambda\left(C_{2}^{2}+C_{3}^{2}\right)}{C_{2}^{2}} a^{3}+\left(C_{1}-2 \lambda\right) a-C_{2}=0
$$

For the special case $\lambda=0$ it is easy to derive the solutions

$$
a=\frac{C_{2}}{C_{1}}, \quad b=\frac{C_{3}}{C_{1}}
$$

and for the special case $\lambda \rightarrow \infty$

$$
a= \pm \frac{C_{2}}{\sqrt{C_{2}^{2}+C_{3}^{2}}}, \quad b= \pm \frac{C_{3}}{\sqrt{C_{2}^{2}+C_{3}^{2}}}
$$

